\title{
Recent Advances and Challenges in Underwater Sensor Networks - Survey
}

\author{
S.Prince Sahaya Brighty \\ Assistant Professor, Department of CSE \\ Sri Ramakrishna Engineering College \\ Coimbatore. \\ Brindha.S.J. \\ II Year, Department of CSE \\ Sri Ramakrishna Engineering College \\ Coimbatore. \\ R.HemaGayathri \\ II Year, Department of CSE \\ Sri Ramakrishna Engineering College \\ Coimbatore.
}

\begin{abstract}
In underwater sensor networks (UWSNs), it is important to determine the location of all the sensors. Localization is the process of estimating the location of each node in a sensor network. There are only few localization schemes for UWSNs even though many localization algorithms have been proposed for terrestrial sensor networks. The characteristics of underwater sensor networks are fundamentally different from that of terrestrial networks. The physical layer environments with rigorous bandwidth limitations are the main characteristic of underwater acoustic channels. A unique set of challenges are posed by the long propagation delays and the variable speed of sound under water for localization in UWSNs. This paper explores the different localization algorithms relevant to underwater sensor networks, and the challenges in meeting the requirements posed by emerging the applications for such networks, likes offshore engineering.
\end{abstract}

KEYWORDS - Localization, Underwater Sensor Networks (UWSN), Positioning.

\section{INTRODUCTION}

In recent trends, the deployment of low cost wireless sensors is proven to be a promising technique for various applications. Underwater applications look into the early warning systems for natural disasters, ecosystem monitoring, oil drilling, and military surveillance. The limited power constraints and processing capability on the sensors makes the management and deployment of wireless sensor networks a greater challenge. The researches related to underwater sensor networks are being done in the recent days. The sensor networks from the physical layer to the application layer have been discussed [9]. Oil drilling generally uses huge exploration vessels that are anchored to the seabed with multiple anchors. These smart sensors can be deployed on the seabed and it can monitor the system parameters and the environmental parameters [11]. These works together with these Remotely Operated Vehicles (ROV), which are controlled from the ship, or Autonomous Underwater Vehicles (AUV), that can autonomously navigate the deep waters are based on some set of instructions. The ROVs/AUVs, sensors and anchors collect and feed information from the seabed. These can measure parameters such as foundation strength and mooring tensions .They also provide accurate position references to the AUVs[5]. The location of the sensors, anchors and the AUVs are needed to be determined in order to deliver data from the seabed to the ship [1]. This problem is especially challenging for deep water applications. Another challenge in underwater acoustic communication is the limited bandwidth long propagation delay, signal failure issue and sensor node failure due to environmental conditions [12]. These nodes must self-configure and it selves to the changes in the ocean environment. Developing efficient underwater communication and networking techniques are involved in the energy conservation of UWSNs [13]. 


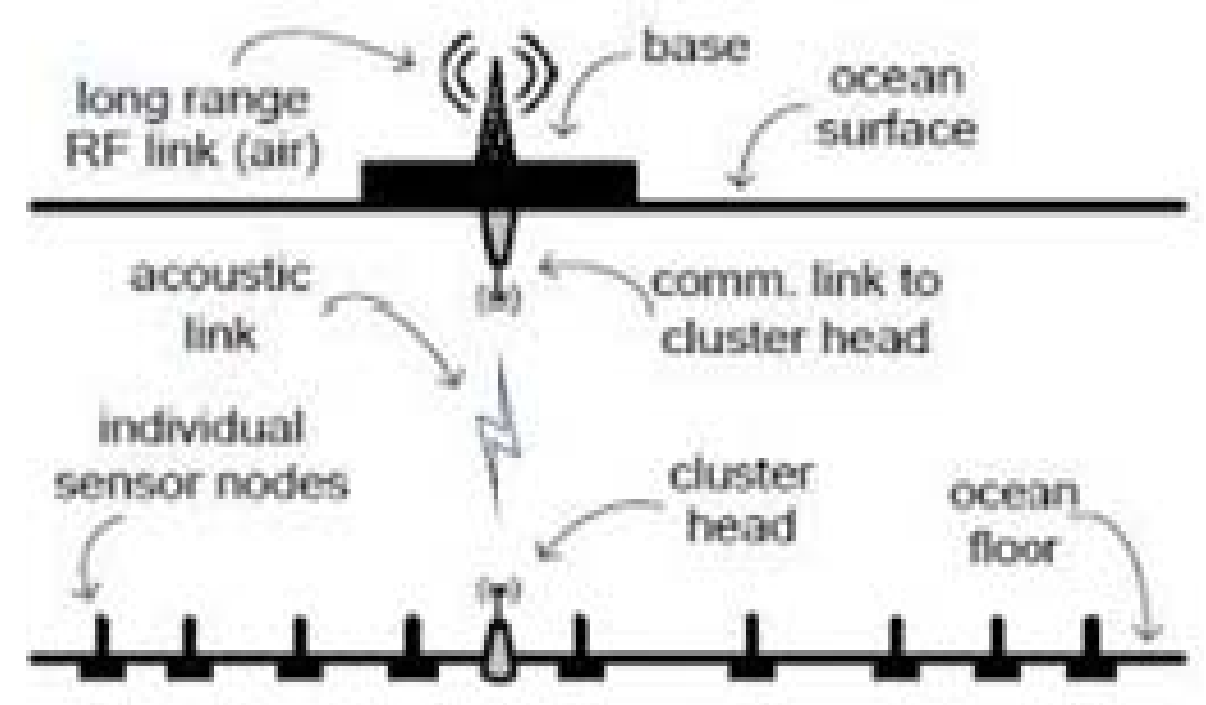

Figure 1. 2D architecture of underwater sensor network.

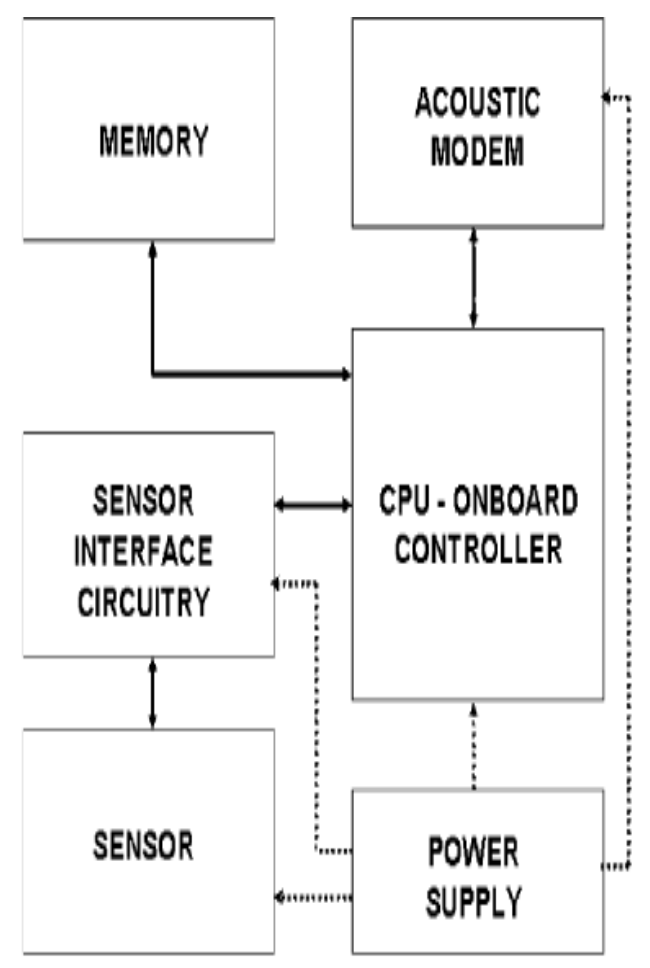

Figure 2. An example application scenario for underwater Sensor Networks.

\section{LOCALIZATION SCHEMES SURVEY}

A sensor's location helps in typically interpreting a sensor network data. For e.g. tracking a moving object, monitoring the physical conditions of some region or reporting the occurrence of certain events [10]. The localization underwater is as challenging as Radio Frequency (RF) waves that are heavily attenuated under water.A number of localization schemes have been proposed that takes into account many factors such as device capability, signal propagation model and energy requirements. The underwater localization involves 
many challenges like node development, node mobility, change in signal strength, time synchronization, variation in sound speed and acoustic channel characteristics [4].

\section{A. Range based scheme}

Range or bearing information like Time Of Arrival (TOA) and Time Difference Of Arrival (TDOA) is used by the range-based localization schemes for position estimation. Locating some nodes in network is known as anchor node or reference node which is used to localize the unlocalized nodes in the networks.

(i) Infrastructure based location schemes: In infrastructure based location schemes, the anchor nodes are deployed on the seabed at pre-determined positions. These are anchor-based. It similar to the GPS scheme. Surface buoys also serve as anchor nodes, whose locations are determined by GPS. The propagation time of the sound signals between the sensor and the anchors are used for computing the distance to multiple anchor nodes. In many cases, the number of independent range measurements exceeds the number of unknown coordinates

(ii) Distributed positioning schemes : In distributed positioning schemes, the positioning infra-structures are not available, i.e., these are anchor-free .Here, the nodes are able to communicate only with their one-hop neighbours and compute the distances to their one-hop neighbours. Distributed positioning algorithms generally have three positioning phases: (a) the distance estimation phase, where nodes estimate the distances to their neighbours (b) the position estimation phase, where a system of linear equations is solved using a least squares approach to estimate the position of the node and (c) a refinement phase, where the accuracy of the algorithm is improved by using an iterative algorithm. This algorithms generally assume anchor nodes to be randomly distributed throughout the network, and the percentage of anchor nodes in the network to be quite high too (520\%) [6].

(iii) Traditional range-based schemes: These schemes uses mobile beacons/anchors whose locations are known

by the fixed anchors. Here the sensor networks are traversed by the mobile beacons while location coordinates of the beacons are contained by the broadcasting beacons. Any node receiving the beacon packet will be able to infer that it must be somewhere near the mobile beacon with a certain probability. RSSI measurements of the received beacon packets are used for ranging purposes. Bayesian inference is used to determine the location of the node, after a number of packets have been received from the mobile beacon.

(iv) Schemes without Anchor/Reference Points: This scheme is different from the other three in that it does not require anchor nodes or beacon signals. In a central server models, the network as a series of equations represent the proximity constraints between nodes, and then uses sophisticated optimization techniques to estimate the location of every node in the network.

\section{B. Range free schemes}

The range-free schemes do not use these range or bearing information,

(i) Hop count based schemes: In hop count based schemes, the anchor nodes are located along the corners of a square grid. This scheme is proposed to improve the accuracy of location estimation when the distribution of node is uniform. DV-Hop is one of the most basic range-free schemes that employ a classical distance vector exchange so that all nodes in the network get distances, in number of hops, to the anchor nodes. Each node maintains a table and exchanges updates only with its neighbours. Once an anchor gets distances to other anchor, it estimates an average distance for one hop, which is then propagated as a correction to the entire network. On receiving the correction, an arbitrary node then estimates its distances to the landmarks (i.e., anchors) in meters, which can be used to perform triangulation [14]. The DV-Hop algorithm performs well only in networks that have uniform and dense node distributions. Schemes like Density-aware Hop-count Localization (DHL) [15] have been proposed to improve the accuracy of location estimation when the node

Distribution in the network is not uniform. This scheme takes into account both the error in distance estimation and the density of a node's neighbourhood when computing the average hop distance that tends to accumulate with the increase of path length.

(ii) Centroid schemes: In centroid schemes, the anchor nodes are placed in such a way that it forms a rectangular mesh. For this scheme to work efficiently, a higher concentration of anchor nodes are required. The anchor nodes send out beacon signals at periodic intervals with their respective locations from which a receiver node infers proximity to a collection of anchor nodes. The location of this node is then estimated to be the centroid of the anchor nodes. Such a scheme would be hard to implement in the underwater context as it would require setting up a rectangular mesh of anchor nodes on the seabed. 
(iii) Area based localization: It is impossible to measure the exact location of every sensor in a large dense wireless sensor networks. The Area Localization Scheme (ALS) and the Appropriate Point in Triangle (APIT) helps in locating the area where the nodes are located. The signal processing or probabilistic schemes uses signal processing techniques for to do the localization [2]. In very large and dense wireless sensor networks, it may not be feasible to accurately measure the exact location of every sensor and furthermore, a coarse estimate of the sensors' locations may suffice for most applications.

(a) Area Localization Schemes (ALS): ALS levels are a centralized range-free scheme that provides an estimation of a sensor's location within a certain area. Anchor nodes send out beacon signals at varying power levels. The grid is divided into multiple smaller areas based on the ranges of the power levels of the anchor nodes. The sensors measure the lowest power level that they receive from each anchor node. This information is then forwarded with the sensor data to the sink for processing. It is represented by an n-dimensional coordinate, where the i'th coordinate that represents the lowest power level from the i'th anchor node.

(b) Appropriate Point in Triangle (APIT) : In this APIT scheme, a node chooses three anchors from all audible anchors (i.e., the anchors from which beacons are received) and tests whether it is inside the triangle region formed by these three anchors. The Point-In-Triangle (PIT) test is the theoretical method used to determine whether a point is inside a triangle or not. The PIT test can be carried out only under ideal physical layer conditions. This can occur only when every node in the network is mobile and can move around its own position. An APIT (Approximate Point in Triangle) test is proposed due to the infeasibility of conducting such a test.

\section{Signal processing/probabilistic schemes}

This scheme uses signal processing techniques for to do the localizations. The U.S. Wireless Corporation fingerprinting scheme [12] falls under this category. It rely on the received signal structure characteristics instead of exploiting signal strength to do localizations [3]. A unique signature has been created for every given location by combining the multi-path pattern with the signal characteristics. This can be achieved by driving a vehicle through the area and acquiring the signal characteristic information. By comparing the received signal characteristic to all the fingerprints in the database, a node's location can be determined. The main drawback of this technique is that a substantial effort is needed for the generation of the signal signature database.

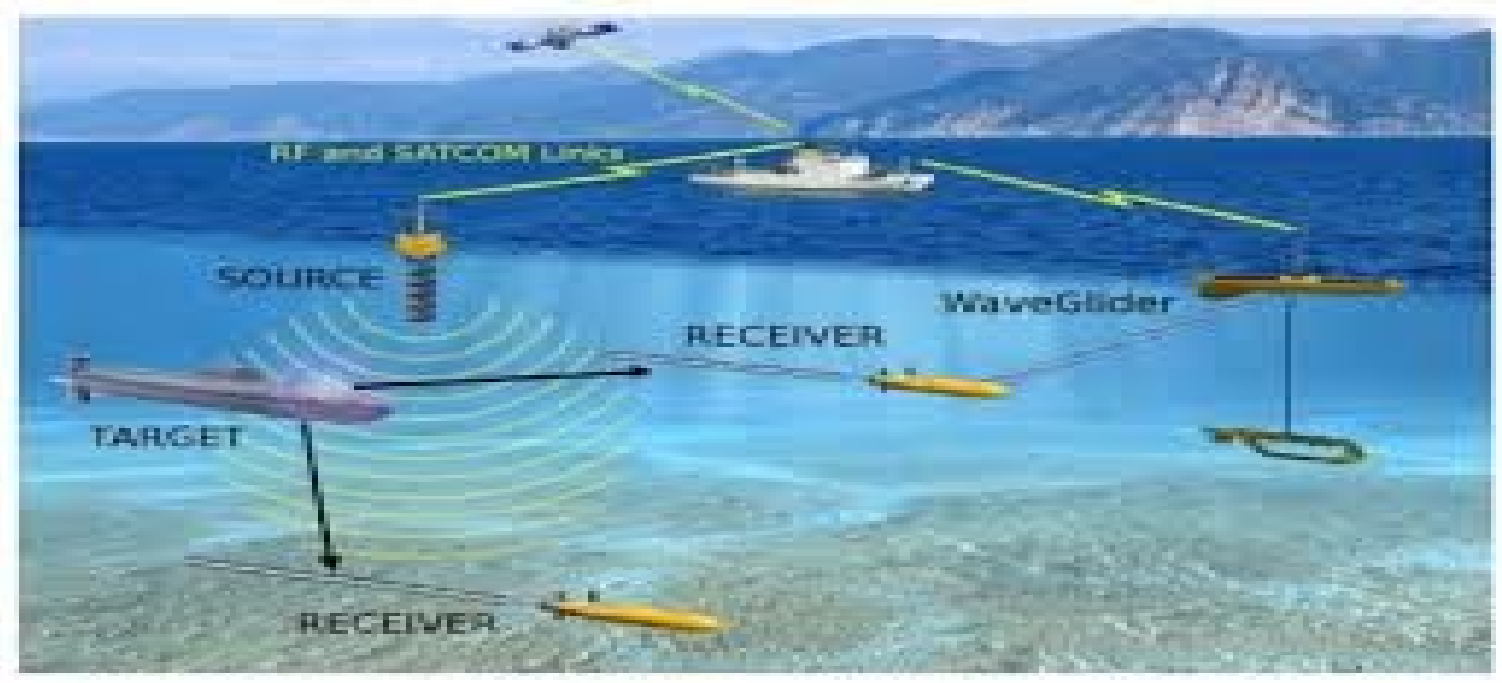

Figure 3. Localization schemes

\section{APPLICATIONS}

Underwater sensor networks have been designed in order to enable applications based on oceanographic data collection, pollution monitoring, mine scouting, offshore exploration, disaster prevention, assisted navigation and tactical surveillance. Exploring natural undersea resources and gathering of scientific data are also been done by sensors [3]. So there arises a need for communications among the underwater devices.

(i) Fastest way for finding underwater information : One of the latest and fastest way of finding information 
Underwater is underwater sensor network. These information are helpful for both human being and also researchers [4].

(ii) Disaster Prevention: This is an important characteristic of underwater sensor network system capable of performing seismic activity that provides tsunami warnings to coastal areas [4].

(iii) Ocean Sampling Networks: In 3D environment, the sensor can be arranged in different depth in the ocean. So that we can sense the ocean area at different depths [5].

(iv) Environmental Monitoring: It is one of the most important applications of UWSN. This includes pollution monitoring, monitoring of ocean currents, improve weather forecasting and so on.

(v) Mine Scouting: The multiple AUVs with acoustic sensor performing simultaneous operations can be used to perform rapid detection of mine like objects.

(vi) Distributed Tactical Surveillance: Fixed underwater sensors and AUVs can collaboratively monitor areas for surveillance, reconnaissance and intrusion detection systems [5].

(vii) Seismic monitoring: This application for underwater Sensor networks is helpful for oil extraction from underwater.

(viii) Equipment Monitoring and Control: This includes monitoring support when the equipment is deployed, possibly associated with tethered power and communications.

The applications of underground sensor networks also include sensing-based cyber physical systems like Collision avoidance, robotic surgery, Nano level manufacturing, air traffic control, healthcare monitoring and so on.

\section{PROBLEMS AND CHALLENGES}

(i) Some sensors use the difference in propagation times of acoustic and radio signals for calculating the distance. This acts as the propagation speed of RF signals in a magnitude of five orders higher than acoustic signals. As the RF waves do not propagate well underwater, the luxury of using RF signals for time synchronization is not available in the underwater scenario.

(ii) In many schemes, the speed of sound is assumed to be a constant. However, the speed of sound depends on the temperature, salinity and depth [11]. Schemes that take into account the variation in the speed of sound are expected to perform better than those that just assume a uniform speed of $1500 \mathrm{~m} / \mathrm{s}$ [7].

(iii) The RSSI-based localization schemes for Under Water Sensor Networks need to take into account multipath effects due to the surface reflection, bottom reflection and backscattering.

(iv) Depending on the roughness, the sea surface can act as a reflector or scatterer of sound. This roughness of the sea surface is dependent on the wave height and the wind speed measured above the sea surface. As like the sea surface, the seabed can also have a reflecting or scattering effect on acoustic waves.

(v) A new research paradigm of Underwater Wireless Sensor Networks (UWSNs) poses challenges like large propagation delays, node mobility and high error probability of acoustic underwater channels, compared to the ground based WSNs. Domingo and Prior (2008) described a protocol named DUCS (Distributed Underwater Clustering Scheme). It is a GPS free routing protocol and it helps in minimizing the exchange of proactive routing message and also it doesn't use the flooding techniques [8].For to eliminate the redundant information it uses the data aggregation.

\section{A. Advantages}

\section{ADVANTAGES AND DISADVANTAGES}

These UWSNs avoids data spoofing and privacy leakage.

(i) It helps in pollution monitoring.

(ii) It also provides early warnings of tsunamis and earthquakes generated undersea [5].

\section{B. Disadvantages}

(i) The sensors also have some drawbacks like limited bandwidth and limited battery power,

(ii) The channel characteristics include long and variable propagation delays.

(iii) It also includes fading problems and high bit error rate.

\section{RELATED WORKS}

(i) 3D wireless networks: Localization in $3 \mathrm{D}$ wireless sensor networks is still under exploration. Only a few researchers have tried to address the problem using both range based and range free techniques. A range 
based technique called "3D - landscape" has been proposed. This scheme addresses the application domain of $3 \mathrm{D}$ terrains. In the scheme, the location - unaware nodes compute their location from the moving location - aware nodes.

(ii) Oceanographic research: Oceanographic researchers have developed underwater sensing and communication systems as like the Ocean Seismic Network program. It developed seismic observatories in the deep ocean, as part of the Global Seismic Network (GSN). GSN has 128 observatories "uniformly" distributed on continents, islands or in the ocean, with a separation distance of $2000 \mathrm{~km}$. Its goal is to monitor a huge area on earth. In contrast, our sensor network covers a much smaller area, and nodes are densely deployed in an ad hoc fashion. Underwater acoustic communication is another related area. Their major focus is the transmission range, bandwidth utilization and reliability with multi-path propagations. There are also experimental and commercial off-the-shelf acoustic modems available today [16]. However, they are designed for long range communications (1-90km), and have weights of over $4 \mathrm{~kg}$. In our hardware design, we focus on short range, low-power modules in a small package. This capability is an enabling factor for long-lived sensor networks.

(iii) Wireless sensor networks: Most work in the sensor network community is focused on terrestrial sensor networks. Virtually all platforms use radio communications. The UC Berkeley motes [15] are based on 8bit microcontrollers and short-range radios. 32bit platforms are normally embedded PCs, such as PC/104s and Star gates. Although the radio propagation in water is very bad, the motes are still used by researchers in marine microorganism monitoring applications. We plan to extend sensor network platforms with a lowpower, short-range acoustic communication device, so that large-scale underwater experiments and applications become possible.

(iv) Underwater networks : There are some prior work in underwater acoustic networking .This research assumes an ad hoc networking model with small to moderate number of nodes. In contrast, our sensor network model consists of hundreds to thousands of nodes, and our application has different requirements. Recently, concurrent with our work, Kong et al.[14] have outlined a research direction in underwater ad hoc networking [22], emphasizing simulation of localization, security, and location-based routing in military applications. Our work instead focuses on prototype hardware and adds time synchronization and other applications.

\section{CONCLUSIONS}

Many new challenges are posed by the problems of UWSNs. This is because of the acoustic transmission medium. The localization schemes and applications of UWSNs are surveyed in this paper. The advantages and disadvantages of UWSNs have been discussed. Also the localization in the application domain of offshore engineering has been discussed.

\section{REFERENCES}

[1] V.Chandrasekhar, W.K.G.Seah ,Yoo Sang Choo and How Won Ee, "Localization in underwater sensor networks - survey and challenges", Proceedings of IEEE conference, Jan2006.

[2] Mukesh Beniwal and Rishipal Singh, "Localization techniques and their challenges in under water wireless sensor networks", Proceedings of International Journal of Computer Science and Information Technologies, Vol 5(3), 2014,4706-4710.

[3] J. Heidemann, Y. Li, A. Syed, J Wills and W. Ye, "Research Challenges and Applications For Underwater Sensor Networking", Proceedings of the IEEE Wireless Communications and Networking Conference (WCNC2006), April 3-6, 2006, Las Vegas, USA.

[4] M. Hahn and J. Rice, "Undersea Navigation via a Distributed Acoustic Communication Network", Proceedings of the Turkish International Conference on Acoustics, July 4-8, 2005, Istanbul, Turkey.

[5] J. Garcia, "Positioning of sensors in Underwater Acoustic Networks", Proceedings of the MTS/IEEE OCEANS Conference, Sep 1923, Washington DC, USA.

[6] Kai Chen,Yi Zhou and Jianhuan He, "A localization scheme of underwater wireless sensor networks", Proceedings of International Journal of Advanced Science and Technology, Vol.4, March 2009.

[7] D. J. Cook and S. K. Das, "Smart environments: technologies, protocols and applications", New York: John Wiley, pp. 13-15, 2004.

[8] K.Sohraby,D. Minoli, and T. Znati,"Wireless sensor networks: technology, protocols and applications," New Jersey: John Wiley, pp. 38-71, 2007.

[9] Q. Ling, Z. Tian, Y. Yin, and Y. Li, "Localized structural health monitoring using energy efficient wireless sensor networks," IEEE Sensors Journal, vol.9, no.11, pp.1596 - 1604, 2009.

[10] I.Akyilidiz et al", Underwater acoustic Sensors networks: research challenges", Ad Hoc Networks Journal.

[11] V.Chandrasekhar and W.K.G.Seah, "Area localization scheme for underwater sensor networks", Proceedings of the IEEE OCEAN Asia Pacific Conference,2006.

[12] Vivek Katiar, Narottam Chand and Naveen Chauhan," Recent advances and future trends in wireless sensor networks", Proceedings of International Journal of Applied Engineering Research, Dindigul, Vol 1(3), 2010.

[13] R. Kay and F. Mattern, "The Design Space of Wireless Sensor Networks", IEEE Wireless $\quad$ Communications Vol.11.No.6 (2004) pp 54-61. 
[14] J. Kong, J. hong Cui, D. Wu, and M. Gerla. Building underwater ad-hoc networks and sensor networks for large scale real-time aquatic applications. In Proceedings of the IEEE MILCOM, Atlantic City, NJ, USA, Oct. 2005.

[15] J. Hill, R. Szewczyk, A. Woo, S. Hollar, D. Culler, and K. Pister. System architecture directions for networked sensors. In Proceedings of the 9th International Conference on Architectural Support for Programming Languages and Operating Systems, pages 93-104, Cambridge, MA, USA, Nov. 2000.

[16] J. Catipovic, D. Brady, and S. Etchemendy. Development of underwater acoustic modems and networks. Oceanography, 6:112-119, Mar. 1993. 